\title{
Prevalencia de Queilitis Actínica en Pescadores Artesanales, Valdivia, Chile
}

\author{
Prevalence of Actinic Cheilitis in Artisanal Fishermen, Valdivia, Chile.
}

Pablo Ríos; Carolina Maldonado²; Paulina Norambuena² \& Manuel Donoso ${ }^{1}$

RÍOS, P.; MALDONADO, C.; NORAMBUENA, P. \& DONOSO, M. Prevalencia de queilitis actínica en pescadores artesanales, Valdivia, Chile. Int. J. Odontostomat., 11(2):192-197, 2017.

RESUMEN: El objetivo de este trabajo consistió en determinar la prevalencia de Queilitis Actínica en pescadores artesanales de la ciudad de Valdivia, Chile. Se realizó un estudio observacional descriptivo de corte transversal durante los meses de noviembre 2014 a abril 2015. La muestra fueron 180 pescadores previamente seleccionados a través de una muestra aleatoria simple, quienes fueron evaluados mediante un examen clínico y además completaron un cuestionario referente a datos personales y hábitos de salud. El 38,8 \% de los pescadores artesanales presentó Queilitis Actínica. El $64,4 \%$ de los pescadores revisados no utiliza protección solar mientras trabaja y todos los casos se ubicaron en el labio inferior. Los pescadores artesanales de la ciudad de Valdivia presentaron una prevalencia de Queilitis Actínica superior a las reportadas a nivel nacional. Nuestros resultados sugieren la implementación de políticas de salud pública centrados en la prevención de Queilitis Actínica en pescadores artesanales de la ciudad de Valdivia.

PALABRAS CLAVE: queilitis actínica, pescadores, lesión pre maligna, prevalencia.

\section{INTRODUCCIÓN}

La Queilitis actínica (QA) es una lesión inflamatoria crónica que afecta el labio inferior en el $95 \%$ de los casos. Cuando tanto el labio superior e inferior son prominentes, el labio superior puede ser más vulnerables a la exposición solar (Vieira et al., 2012). Se manifiesta a través de desecación y/o erosión de la mucosa labial y pérdida del borde bermellón del labio. Su principal factor etiológico es la exposición a la radiación solar ultravioleta (RUV), especialmente la tipo $B$, debido a su mayor poder de penetración en comparación a la tipo A (Lopes et al., 2015). Los factores de riesgo asociados son el tabaquismo, personas de piel clara y personas que trabajan al aire libre (de Oliveira Ribeiro et al., 2014). La historia natural de la QA puede seguir cualquiera de las siguientes opciones: regresión espontánea de la lesión, permanecer sin cambios a través del tiempo o evolucionar a un carcinoma espinocelular (CEC) invasivo. Se ha reportado una tasa de regresión espontánea de la lesión de entre un $15 \%$ a un $55 \%$ en un periodo de un año (Harvey et al., 1996). Sin embargo, el porcentaje de casos de QA que evoluciona en un CEC invasivo no se conoce con certeza. En un estudio prospectivo de 12 meses, se determinó una incidencia de transformación maligna de QA en CEC invasivo de un 0,24 $\%$ (Marks et al., 1986). No obstante, se ha determinado que el riesgo de progresión maligna en 10 años sería de un $6,1 \%$ a un 10,2 \% (Trakatelli et al., 2007) incrementándose a un $40 \%$ en pacientes inmunocomprometidos (Fuchs \& Marmur, 2007). Se debe mencionar además, que sobre el $80 \%$ de los casos de CEC invasivos ubicados en zonas expuestas a la RUV se desarrollan en relación a una lesión de QA preexistente (Ferrándiz et al., 2014). En Chile, existen inscritos más de noventa mil pescadores artesanales, de los cuales el $5 \%$ se registra en la ciudad de Valdivia (Servicio Nacional de Pesca y Acuicultura, 2014). Debido a su naturaleza premaligna (Fuchs \& Marmur) y considerando que el CEC de labio representa un $20 \%$ de los carcinomas orales, es necesario establecer la prevalencia de QA en pescadores artesanales para evaluar la implementación de políticas públicas que incluyan programas de promoción, prevención y diagnóstico temprano de QA en

\footnotetext{
${ }^{1}$ Instituto de Odontoestomatología, Facultad de Medicina, Universidad Austral de Chile, Valdivia, Chile.

${ }^{2}$ Escuela de Odontología, Facultad de Medicina, Universidad Austral de Chile, Valdivia, Chile.
} 
poblaciones de riesgo, fomentando el autocuidado y a su detección temprana por parte de los profesionales de la salud.

En la actualidad, no existen datos certeros sobre la prevalencia de QA en Chile. Investigaciones previas han reportado valores que varían desde el 0,9\% al 43 \% (Nicolini et al., 1989; Espinoza et al., 2003). En la ciudad de Valdivia no existen datos previos de prevalencia de QA en pescadores artesanales. Por lo tanto, el objetivo de este estudio fue determinar la prevalencia de QA en pescadores artesanales de la ciudad de Valdivia, Chile.

\section{MATERIAL Y MÉTODO}

Se realizó un estudio observacional descriptivo de corte transversal entre noviembre del año 2014 y abril del año 2015 en la ciudad de Valdivia, Chile. El reporte se hizo de acuerdo a las recomendaciones entregadas por la pauta STROBE (Vandenbroucke et al., 2008). Antes de dar inicio a la investigación, se solicitó aprobación al Comité de Ética del Servicio de Salud de Valdivia (ORD No 433) y autorización por medio de la firma de un consentimiento informado a cada pescador examinado. La población objetivo fueron los pescadores artesanales de Valdivia. Según el registro de SERNAPESCA, para el año 2014 en la ciudad de Valdivia, se determinó que la población elegible fue de 1.153 individuos Valdivia (Servicio Nacional de Pesca y Acuicultura). El tamaño de la muestra se calculó en base a un nivel de confianza del $95 \%$ y una prevalencia esperada del evento de 16,6 \% (Orozco et al., 2013), dando como resultado un $n=180$ pescadores. Se realizó un muestreo aleatorio simple para seleccionar a los participantes. Para el cálculo de tamaño de la muestra se utilizó el programa online del Instituto Datakey (Instituto datakey, 2014). Se incluyeron en este estudio los pescadores pertenecientes a la comuna de Valdivia que aceptaron participar. Los pescadores que padecieron alguna enfermedad infecto-contagiosa al día del examen fueron excluidos.

Dos examinadores previamente calibrados (Kappa 0,85) realizaron examen clínico de la región perioral de cada uno de los individuos. Este examen se realizó ubicando al individuo sentado frente al examinador, utilizando inspección e iluminación directa. Durante la examinación clínica se evaluaron las siguientes características clínicas: sequedad (se consideró aquellos labios con falta de humedad), esfumación del borde del bermellón del labio (se registraron aquellos casos en que había pérdida de continuidad entre el borde del bermellón y piel de forma total o parcial), atrofia (se registró en aquellos casos donde había un adelgazamiento del epitelio a nivel de la mucosa), pliegues labiales (se consideraron los casos en que hubiera pérdida de continuidad en sentido vertical), lesiones escamosas (se registraron aquellas capas de tejido que se desprenden, a diferencia de la costra y que se ubicaban a nivel del bermellón del labio), aumento de volumen (se consideró aquellos casos donde se veía un aumento de volumen que se encontraba asociado a una pérdida de elasticidad del labio), costras (aquellas lesiones que estaban con formación de tejido de cicatrización, tejido que no se desprendía a diferencia de las lesiones escamosas), ulceración (lesiones del labio en la cual había pérdida de sustancia) y síntomas como ardor o prurito (registrados al momento de la anamnesis). Se consideró presencia de QA en aquellos pescadores que presentaron esfumación del borde bermellón del labio, asociado a una o más de las características clínicas mencionadas.

La información fue almacenada en una base de datos, asegurando su confidencialidad. A partir de la información obtenida de los cuestionarios se analizaron las siguientes variables: género, edad, años de exposición solar, uso de medidas de protección solar, consumo de tabaco y alcohol. Los individuos fueron clasificados como ex fumador, no fumador y fumador incluyendo en este último la cantidad de cigarrillos fumados diariamente. El consumo de alcohol se clasificó en base al consumo diario o semanal declarado en el cuestionario.

Los datos se tabularon con la ayuda de un formulario electrónico de Google Drive y se analizaron mediante estadística descriptiva con el software SPSS (SPSS para Windows ${ }^{\circledR} 20$, SPSS Inc., Chicago, III. USA).

\section{RESULTADOS}

Se examinaron 180 pescadores artesanales, de los cuales 177 (98,3\%) fueron hombres. Del total de pescadores examinados, 70 (38,8 \%) presentaron QA $(95 \% \mathrm{Cl}=32,2-46,7)$. Al momento del diagnóstico, $31(44,2 \%)$ pescadores presentaron sintomatología (prurito). La totalidad de los casos se presentaron en el labio inferior, el $82,85 \%(n=58)$ de los casos tiene 
RíoS, P.; MALDONADO, C.; NORAMBUENA, P. \& DONOSO, M. Prevalencia de queilitis actínica en pescadores artesanales, Valdivia, Chile. Int. J. Odontostomat., 11(2):192-197, 2017.

Tabla I. Frecuencias de variables estudiadas.

\begin{tabular}{|c|c|c|c|c|c|}
\hline \multirow[b]{2}{*}{ Variables } & \multicolumn{2}{|c|}{$\begin{array}{c}\text { Presencia de Queilitis } \\
\text { Actínica }\end{array}$} & \multicolumn{2}{|c|}{$\begin{array}{c}\text { Ausencia de } \\
\text { Queilitis Actínica }\end{array}$} & \multirow{2}{*}{$\begin{array}{c}\text { Tota } \\
\mathrm{n}\end{array}$} \\
\hline & $\mathrm{n}$ & $\%$ & $\mathrm{n}$ & $\%$ & \\
\hline \multicolumn{6}{|l|}{ Género } \\
\hline Femenino & 0 & 0 & 3 & 100 & 3 \\
\hline Masculino & 70 & 39,54 & 107 & 60,45 & 177 \\
\hline \multicolumn{6}{|l|}{ Edad } \\
\hline 20-29 años & 6 & 15,78 & 32 & 84,21 & 38 \\
\hline 30-39 años & 6 & 18,18 & 27 & 81,81 & 33 \\
\hline 40-49 años & 30 & 51,72 & 28 & 48,27 & 58 \\
\hline 50 o más & 28 & 54,90 & 23 & 45,09 & 51 \\
\hline \multicolumn{6}{|c|}{ Exposición solar acumulada } \\
\hline 0-9 años & 9 & 27,27 & 24 & 72,72 & 33 \\
\hline 10-19 años & 10 & 20 & 40 & 80 & 50 \\
\hline Más de 20 años & 51 & 52,57 & 46 & 47,42 & 97 \\
\hline \multicolumn{6}{|c|}{ Medidas de protección solar } \\
\hline Bloqueador solar & 10 & 25,64 & 29 & 74,35 & 39 \\
\hline Sombrero & 3 & 21,42 & 11 & 78,57 & 14 \\
\hline Ambos & 0 & 0 & 11 & 100 & 11 \\
\hline Ninguno & 57 & 49,13 & 59 & 50,86 & 116 \\
\hline \multicolumn{6}{|l|}{ Hábito de fumar } \\
\hline $\mathrm{Si}$ & 43 & 38,05 & 70 & 61,94 & 113 \\
\hline No & 22 & 40,74 & 32 & 59,25 & 54 \\
\hline Ex fumador & 5 & 38,46 & 8 & 61,53 & 13 \\
\hline \multicolumn{6}{|c|}{ Consumo de alcohol } \\
\hline $\mathrm{Si}$ & 12 & 66,66 & 6 & 33,33 & 18 \\
\hline No & 58 & 35,80 & 104 & 64,19 & 162 \\
\hline
\end{tabular}

una edad mayor a 40 años, con una exposición solar acumulada en el $52,57 \%(n=51)$ de los casos mayor a 20 años. El 64,4\% $(n=116)$ de los pescadores revisados no utilizaba protección solar mientras navegaba. De los entrevistados el 62,7 \% ( $n=113)$ relató ser fumador, dentro de los cuales el $38 \%(n=43)$ presentó QA, según se observa en la Tabla I.

\section{DISCUSIÓN}

Este estudio tuvo por objetivo determinar la prevalencia de QA en pescadores artesanales de la ciudad de Valdivia, Chile. La prevalencia de QA en la ciudad de Valdivia fue de un $38,8 \%$; lo cual difiere a lo reportado por estudios previos realizados en otras ciudades de Chile. Espinoza et al., determinaron que la prevalencia de QA en la ciudad de Santiago fue de 0,9\%, Orozco et al. reportaron una prevalencia de $16,6 \%$ en la ciudad de Talca y en la ciudad Valparaíso, Fuentes et al. (2012) reportaron una prevalencia de 22,5\%. Estas diferencias en la prevalencia de la enfermedad pueden explicarse por varios factores, tales como el sub-diagnóstico de la patología, ubicación geográfica, tipo de población estudiada, así como los criterios utilizados para el diagnóstico de la enfermedad (Nicolini et al.; Orozco et al.). Aquellos pescadores diagnosticados con QA fueron tratados con terapia farmacológica y derivados a la Unidad de Patología Maxilofacial del Hospital Regional de Valdivia.

Se determinó una alta prevalencia $(82,8 \%)$ de QA en pescadores mayores de 40 años. Datos previos desde una muestra chilena, indican que los pacientes sobre 45 años presentan una mayor incidencia. La edad de manifestación fue mayoritariamente en la quinta década de edad, lo que se condice con publicaciones previas (de Souza Lucena et al., 2012). El 52,5\% de los pescadores con QA presentó una exposición solar acumulada mayor a 20 años. Por lo tanto, hay un efecto acumulativo de estas agresiones, y cuanto mayor es el individuo, mayor es la probabilidad de desarrollar QA (de Souza Lucena et al.). Resultados publicados previamente muestran que los individuos que se exponen habitualmente al sol desde edades tempranas, y con una historia de exposición solar acumulada durante su vida, los hacen más susceptibles de desarrollar QA (Espinoza et al.; Orozco et al.). 
El $64,4 \%$ de los pescadores examinados relató no utilizar medidas de protección solar, lo cual es un factor de riesgo debido a que el factor etiológico predominante de QA es la exposición a largo plazo a la RUV. Por esta razón se sugiere utilizar medidas generales de prevención para reducir la exposición al sol, estas incluyen evitar la luz solar cuando la RUV es más intensa (10 a.m. a 4 p.m.); cubriendo la piel expuesta, llevar un sombrero que cubra el cuello, la cara y las orejas y el uso de un protector solar (Huber, 2010). No obstante, a pesar que la RUV es considerada un factor etiológico para el desarrollo de QA, no existe evidencia que soporte la hipótesis de que la RUV genere una mayor probabilidad de malignización. En ciudades situadas en latitudes más bajas, la exposición a la RUV es alta, lo que hace a la población más susceptible al desarrollo de QA (Martins-Filho et al., 2011). La ciudad de Valdivia presenta un índice de RUV promedio de 1,4 considerándose una zona de baja exposición (Instituto de Ciencias Físicas y Matemáticas, 2015). Esto no es congruente con la alta prevalencia de QA hallada en nuestro estudio. Una posible explicación a este hecho es la alta movilidad que presentan los pescadores inherente a su labor, lo que implica desplazamientos por distintos puntos geográficos $\mathrm{y}$, por ende, distintos niveles de exposición a la RUV. Existe evidencia que el uso regular de protección solar puede ser efectivo en prevenir la incidencia de QA en la comunidad en general y en la prevención de cáncer de piel (Darlington et al., 2003).

El $38 \%$ de los pescadores diagnosticados con QA se declararon fumadores activos. Según PiñeraMarques et al. (2010) el estado de la enfermedad puede ser agravado en los fumadores, porque la exposición continua al calor generado por la combustión de humo puede acelerar el proceso de la carcinogénesis de labios en los individuos que son también expuestos a la radiación solar. Sin embargo, nuestro estudio no encontró una mayor prevalencia de QA en fumadores y tampoco se encontró una relación entre los pescadores que fumaron más de 20 cigarros, versus los que fumaban menos de 10 cigarros al día con aumento de $\mathrm{QA}$.

El $20,5 \%$ de los pescadores que presentaron QA relató consumir alcohol frecuentemente. Aún no existe consenso respecto de la influencia del alcohol en el desarrollo de QA. Campisi \& Margiotta determinaron una asociación estadísticamente significativa entre consumo de alcohol y presencia de QA. Sin embargo, de Souza Lucena no reportó asociación entre ambos factores.
El diagnóstico diferencial de QA abarca un amplio espectro de patologías tales como el CEC, carcinoma de células basales (CCB), melanoma amelanótico, lupus eritematoso discoide, liquen plano oral, queilitis glandular, queilitis angular, queilitis granulomatosa y cáncer metastásico (Jadotte \& Schwartz, 2012). En la actualidad, variadas técnicas han demostrado ser efectivas para realizar el diagnóstico diferencial. La dermoscopía puede ayudar a distinguir la QA de un CCB. A su vez, el microscopio láser confocal de barrido ha demostrado tener una buena sensibilidad y especificidad en el diagnóstico de QA (Ferrándiz et al.). Sin embargo, estas técnicas son utilizadas principalmente con fines de investigación más que para exámenes clínicos rutinarios. En nuestra investigación, no utilizamos estas técnicas ya que, en base a la evidencia actual, el diagnóstico de QA es eminentemente clínico (Huber; Ferrándiz et al.). Sin embargo, en aquellos casos en los cuales se sospecha de un proceso de transformación maligna se recomienda la realización de un biopsia para su examen histopatológico (Huber; Jadotte \& Schwartz). Esta fue la principal limitación del estudio ya que, en algunos casos, pudo existir sub-diagnóstico o sobre diagnóstico de la lesión.

Se presentaron sesgos de memoria, debido a que los participantes no recordaban con exactitud sus respuestas a las variables encuestadas y también sesgo de respuesta por parte del entrevistado ya que los encuestados respondían en base a lo que ellos consideraban correcto y no reflejaba su real condición.

En Chile se estima que la mortalidad por cáncer oral y faríngeo corresponde aproximadamente al $1,6 \%$ del total de todos los cánceres (Guzmán et al., 2011). EI CEC de labio representa cerca del $20 \%$ de los carcinomas orales, siendo más común en hombres y con un peak de ocurrencia entre la sexta y séptima década, además, es más prevalente en el labio inferior que en el superior. Según un estudio realizado, la sobrevida a los cinco años para el CEC de labio en Chile fue aproximadamente $73 \%$, distinta a lo reportado en EE.UU por las cifras SEER (Surveillance, Epidemiology and End Results) del Instituto Nacional del Cáncer, la cual corresponde a un 89,5 \% (Cabello et al., 2015). Estas diferencias pueden explicarse por la desinformación de la población frente a este tipo de cáncer. Salihu et al. (2014) mencionan que la principal razón de un diagnóstico y tratamiento tardío de un CEC de labio es el desconocimiento de la población del riesgo inherente de una lesión oral. El tratamiento oportuno del CEC de labio inferior es importante ya que la 
mortalidad asociada a esta patología es alta si no es tratada. Tomando en cuenta la naturaleza premaligna de la QA y la alta prevalencia en pescadores, creemos que es necesario implementar políticas de salud pública que aporten información relevante a los pescadores con el fin de favorecer la pesquisa precoz de $\mathrm{QA}$, fomentando la promoción y prevención.

\section{AGRADECIMIENTOS}

Esta investigación está basada en la tesis de Carolina Maldonado y Paulina Norambuena, la cual es requisito para optar al título de Cirujano Dentista en la Escuela de Odontología de la Universidad Austral de Chile. Agradecimientos por el apoyo financiero de la Escuela de Odontología Universidad Austral de Chile. El manuscrito no presenta conflictos de intereses por parte de los autores.

RÍOS, P.; MALDONADO, C.; NORAMBUENA, P. \& DONOSO, M. Prevalence of actinic cheilitis in artisanal fishermen, Valdivia, Chile. Int. J. Odontostomat., 11(2):192-197, 2017.

ABSTRACT: The aim of this study was to determine the prevalence of actinic cheilitis in fishermen in the city of Valdivia, Chile 2015. A descriptive observational crosssectional study was realized from November 2014 to April 2015. The sample included 180 fishermen, previously selected through a simple random sample. The subjects were evaluated by clinical examination and also completed a questionnaire on personal data and health habits. Of the total fishermen surveyed $38.8 \%$ presented Actinic cheilitis. Furthermore, $64.4 \%$ of the fishermen interviewed reported they do not use sun protection while working and all cases were located in the lower lip. The fishermen in Valdivia showed a higher prevalence of Actinic cheilitis than that found nationally. Our results suggest the implementation of public health policies focusing on the prevention of actinic cheilitis in artisanal fishermen in the city of Valdivia.

KEY WORDS: actinic cheilitis, fishworkers, premalignant lesion, prevalence.

\section{REFERENCIAS BILIOGRÁFICAS}

Cabello, B. T.; Sazo, B. N.; Salgado, F. A. \& Martínez, R. B. Sobrevida en carcinoma espinocelular de labio. Rev. Med. Chile, 143(7):84755, 2015.

Darlington, S.; Williams, G.; Neale, R.; Frost, C. \& Green, A. A randomized controlled trial to assess sunscreen application and beta carotene supplementation in the prevention of solar keratoses. Arch. Dermatol., 139(4):451-5, 2003.

de Oliveira Ribeiro, A.; da Silva, L. C. \& Martins-Filho, P. R. Prevalence of and risk factors for actinic cheilitis in Brazilian fishermen and women. Int. J. Dermatol., 53(11):1370-6, 2014.

de Souza Lucena, E. E.; Costa, D. C.; da Silveira, E. J. \& Lima, K. C. Prevalence and factors associated to actinic cheilitis in beach workers. Oral Dis., 18(6):575-9, 2012

Espinoza, I.; Rojas, R.; Aranda, W. \&, Gamonal, J. Prevalence of oral mucosal lesions in elderly people in Santiago, Chile. J. Oral Pathol. Med., 32(10):571-5, 2003.

Ferrándiz, C.; Fonseca-Capdevila, E.; García-Diez, A.; GuillénBarona, C.; Belinchón-Romero, I.; Redondo-Bellón, P.; MorenoGiménez, J. C. \& Senán, R. Spanish adaptation of the European guidelines for the evaluation and treatment of actinic keratosis. Actas Dermosifiliogr., 105(4):378-93, 2014.

Fuchs, A. \& Marmur, E. The kinetics of skin cancer: progression of actinic keratosis to squamous cell carcinoma. Dermatol. Surg., 33(9):1099-101, 2007.

Fuentes, C. R.; González, A. W.; Ajudarte, L. M.; Ahumada, S. K.; Acuña, O. M. \& Campos, M. N. Prevalencia de lesiones cutáneas asociadas al sol y queilitis actínica en pescadores de Caleta Portales y Caleta El Membrillo de Valparaíso, Chile. Rev. Fac. Odontol. Univ. Valpso., 4(3):1253-8, 2012.

Guzmán, G. P.; Villaseca, H. M.; Antonio, P. L.; Araya, O. J.; Aravena, M. P.; Cravero, P. C.; Pino, M. P. \& Roa, S. J. Carcinoma epidermoide oral y orofaringeo: Estudio clinico-patológico. Rev. Chil. Cir., 63(3):250-6, 2011.

Harvey, I.; Frankel, S.; Marks, R.; Shalom, D. \& Nolan-Farrell, M. Non-melanoma skin cancer and solar keratoses. I. Methods and descriptive results of the South Wales Skin Cancer Study. Br. J. Cancer, 74(8):1302-7, 1996.

Huber, M. A. White oral lesions, actinic cheilitis, and leukoplakia: confusions in terminology and definition: facts and controversies. Clin. Dermatol., 28(3):262-8, 2010.

Instituto datakey. Utilidades para el Cálculo del Tamaño Muestra y Realización del Muestreo. San Sebastián, Instituto datakey, 2014. Disponible en: http://www.datakey.galeon.com/muestra.html

Instituto de Ciencias Físicas y Matemáticas. Monitoreo Índice UV Valdivia - Región de Los Ríos. Valdivia, Instituto de Física, Facultad de Ciencias, Universidad Austral de Chile, 2015. Disponible en http://www.radiacionuv.cl

Jadotte, Y. T. \& Schwartz, R. A. Solar cheilosis: an ominous precursor part II. Therapeutic perspectives. J. Am. Acad. Dermatol., 66(2):187-98, 2012.

Lopes, M. L.; Silva Júnior, F. L.; Lima, K. C.; Oliveira, P. T. \& Silveira, É. J. Clinicopathological profile and management of 161 cases of actinic cheilitis. An. Bras. Dermatol., 90(4):505-12, 2015.

Marks, R.; Foley, P.; Goodman, G.; Hage, B. H. \& Selwood, T. S. Spontaneous remission of solar keratoses: the case for conservative management. Br. J. Dermatol., 115(6):649-55, 1986.

Martins-Filho, P. R.; Da Silva, L. C. \& Piva, M. R. The prevalence of actinic cheilitis in farmers in a semi-arid northeastern region of Brazil. Int. J. Dermatol., 50(9):1109-14, 2011.

Nicolini, S.; Ascorra, C.; Guzman, C. \& Latife, A. V. Actinic cheilitis in Quinta fishing workers: prevalence and associated histopathological aspects. Odontol. Chil., 37(1):169-74, 1989.

Orozco, P.; Vásquez, S.; Venegas, B. \& Rivera, C. Prevalencia de queilitis actínica en trabajadores expuestos a radiación ultravioleta en Talca, Chile. Rev. Clin. Periodoncia Implantol. Rehabil., 6(3):127-9, 2013.

Piñera-Marques, K.; Lorenço, S. V.; da Silva, L. F. F.; Sotto, M. N. \& Carneiro, P. C. Actinic lesions in fishermen's lower lip: clinical, cytopathological and histopathologic analysis. Clinics, 65(4):3637, 2010.

Salihu, S.; Güven, O.; Gllareva, E.; Prekazi, M. \& Salihu, L. A clinical study on survival rate of patients with squamous cell carcinoma 
RíOS, P.; MALDONADO, C.; NORAMBUENA, P. \& DONOSO, M. Prevalencia de queilitis actínica en pescadores artesanales, Valdivia, Chile. Int. J. Odontostomat., 11(2):192-197, 2017.

of the lower lip in Kosovo. J. Craniomaxillofac. Surg., 42(8):17737, 2014.

Servicio Nacional de Pesca y Acuicultura. Registro Pesquero Artesanal del Servicio Nacional de Pesca y Acuicultura. Santiago de Chile, Servicio Nacional de Pesca y Acuicultura, Gobierno de Chile, 2014. Disponible en: http://www.sernapesca.cl/ index.php?option=com_content\&task=view\&id=84\&ltemid=222

Trakatelli, M.; Ulrich, C.; del Marmol, V.; Euvrard, S.; Stockfleth, E. \& Abeni, D. Epidemiology of nonmelanoma skin cancer (NMSC) in Europe: accurate and comparable data are needed for effective public health monitoring and interventions. Br. J. Dermatol., 156 Suppl. 3:1-7, 2007

Vandenbroucke, J. P.; Von Elm, E.; Altman, D. G.; Gøtzsche, P. C.; Mulrow, C. D.; Pocock, S. J.; Poole, C.; Schlesselman, J. J. \& Egger, M. Mejorar la comunicación de estudios observacionales en epidemiología (STROBE): explicación y elaboración. Gac. Sanit., 23(2):158e1-28, 2008.

Vieira, R. A.; Minicucci, E. M.; Marques, M. E. \& Marques, S. A. Actinic cheilitis and squamous cell carcinoma of the lip: clinical, histopathological and immunogenetic aspects. An. Bras. Dermatol., 87(1):105-14, 2012.

\author{
Dirección para correspondencia \\ Pablo Ríos, Profesor Asociado \\ Instituto de Odontoestomatología \\ Facultad de Medicina \\ Universidad Austral de Chile \\ Rudloff \#1640 \\ Valdivia \\ CHILE \\ E-mail: pablo.rios.uach@gmail.com
}

Recibido : 14-03-2017

Aceptado: 13-07-2017 